\title{
Why is the radial flow in central pA collisions stronger than in AA?
}

\author{
Tigran Kalaydzhyan and Edward Shuryak \\ Department of Physics and Astronomy \\ Stony Brook University \\ Stony Brook NY 11794 USA
}

\begin{abstract}
Both the transverse size and entropy density per area in central $p A$ collisions is smaller than in central AA, and yet the radial flow is stronger. We propose an explanation to this puzzle. Using a weak attraction between strings through the $\sigma$-meson exchange, fitted to the lattice data, we find collective implosion of the "spaghetti" multi-string state. Colelctivization of the sigma field of the strings is the QCD analogue of the black hole formation occurring in holographic models.
\end{abstract}

Keywords: QGP, QCD strings, radial flow

\section{Introduction}

AdS/CFT correspondence has lead to development of various models, collectively known as AdS/QCD, aiming to describe strong coupling regime of QCD. Formation of the QGP fireball in this approach corresponds to black hole formation in 5-dimensional space. The result of multiple studies show a very rapid transition from a non-equilibrium stage to hydrodynamical expansion, in accord with observations for heavy ion collisions.

In contrast to that, theory of $p p$ and $p A$ collisions mostly developed inside the perturbative framework, resulting e.g. in BFKL description of the Pomeron, amended by studies of the saturation phenomena. Recently a holographic model of the Pomeron has been developed in Ref.[1], connecting "hard" perturbative regime with a "soft" one, in terms of production of (two) QCD strings. Crucial observation made in Ref. [2] is that due to cylindrical geometry of the produced string worldline, the Pomeron problem can be described by an effective temperature, related to the impact parameter $b$, even in elementary minimally biased pp collisions. In particular it was shown in [2] that perturbative regime at small $b$ and "stringy" regime at large $b$ are separated by the third "Hagedorn" regime, in which strings are born in thermally highly excited state. Such objects - known as "self-interacting string balls" - has been recently studied in our paper [3], in specialized lattice simulations.

These recent developments provided a background for the present study, devoted to central $p A$ collisions. While each individual NN interaction in $p A$ collisions happens in the large $b$ "stringy" regime of the Pomeron, the total number of them is large $\sim 20$, leading to large number of strings $\sim 40$ or more, in the so called "spaghetti" configuration. The question we address below is what are the conditions under which such system undergoes a "collapse" into the black-hole-like state.

But before we do so, let us elucidate a puzzle recently discovered experimentally: the radial flow in central $p A$ collisions seem to be stronger than in central $A A$, while the entropy density per transverse area show a different systematics

$$
\frac{d N_{\text {maximal }}^{\text {pA }}}{d A_{\perp}} \sim \frac{d N_{\text {peripheral }}^{A A}}{d A_{\perp}}<\frac{d N_{\text {central }}^{A A}}{d A_{\perp}}<\frac{d N_{\text {maximal }}^{\text {pp }}}{d A_{\perp}}
$$


The simplest estimates of the initial entropy density in various collisions use as an input the total multiplicity and the transverse area of the collision. For pA the initial transverse area can be estimated as $A_{\perp} \approx \sigma_{N N} \approx 10 \mathrm{fm}^{2}$. If so, it leads to entropy density comparable to peripheral $A A$ and - as we will show below - to a radial flow, comparable to that in peripheral $A A$ but much weaker than observed in central $p A$.

\section{The radial flow and the size of the fireball}

The best signature of the radial flow is the so-called $m_{\perp}=\sqrt{m^{2}+p_{\perp}^{2}}$ slope, $T^{\prime}$, defined by the fit $\frac{d N}{d y d m_{\perp}^{2}} \sim$ $\exp \left(-\frac{m_{\perp}}{T^{\prime}}\right)$. A regime in which $T^{\prime}$ is the same for all secondaries is called the " $m_{\perp}$-scaling": it indicates an independent string fragmentation. A regime in which $T^{\prime}$ grows approximately linearly with the particle mass indicates the presence of a collective flow. A sample of such slopes for pA collisions recently measured by CMS [4] and ALICE [5] display a transition between those two regimes. In Fig. 1(a) the bullet points show $T^{\prime}$ for pions, kaons and protons for the highest multiplicity bin (235 tracks in the CMS definition). As predicted a few months before the experiment in the first version of the paper [6], the pA slopes exceeding those of central PbPb LHC collisions, previously record-holding on the radial flow, were observed.

Hydrodynamics provides a connection between the initial and the final properties of the system, and one should eventually finds out what the initial size is, provided the hydro works. For the problem at hand - to see how the result depends on the size of the system - it is convenient to follow the paper of Zahed and one of us [6], in which the radial flow is studied with the use of the (azimuthal angle and rapidity independent) Gubser's solution [7]. From the proper time and transverse radius $\bar{\tau}, \bar{r}$ (with the bar) we proceed to dimensionless variables $t=q \bar{\tau}, r=q \bar{r}$ by the scaling factor $q$, the first parameter of the model. The solution for the transverse velocity and the energy density reads

$$
v_{\perp}(t, r)=\frac{2 t r}{1+t^{2}+r^{2}}, \quad \frac{\epsilon}{q^{4}}=\frac{\hat{\epsilon}_{0} 2^{8 / 3}}{t^{4 / 3}\left[1+2\left(t^{2}+r^{2}\right)+\left(t^{2}-r^{2}\right)^{2}\right]^{4 / 3}} .
$$

The energy density has a second dimensionless parameter $\hat{\epsilon}_{0}$ related to the multiplicity $d N / d y$.

In Fig. 1(b) we show freezeout curves. The blue line marked AA corresponds to the central PbPb collisions at LHC, its parameters $q=1 / 4.3 \mathrm{fm}, \hat{\epsilon}_{0}=2531, T_{f}=120 \mathrm{MeV}$ is our benchmark. Two thin black lines are for the pPb case, they both have $T_{f}=170 \mathrm{MeV}$ and the same multiplicity but different sizes, to be referred as "large" $q=1 / 1.6 \mathrm{fm}$ for the lower dotted line, and "twice compressed" one with $q=1 / 0.8 \mathrm{fm}$ for the upper thin solid line. The arrow indicates a transition between them. The "large" one has freezeout, in such scaled coordinates, well below the AA benchmark, and its radial flow is respectively weaker. The latter is above it, and its radial flow is stronger than in AA. The maximal transverse velocities on these three freezeouts are, respectively, $v_{\perp}^{p A_{i}}=0.56<v_{\perp}^{A A}=0.81<v_{\perp}^{p A_{f}}=0.84$. The upper red line is our guess for the maximal multiplicity pp collisions, assuming its $q=1 / 0.5 \mathrm{fm}$, shown for comparison: it has even stronger radial flow with maximal $v_{\perp}^{p p}=0.93$. We thus conclude that the initial size of the pA system seems to be reduced, compared to the naive estimates above.

\section{String interaction via the $\sigma$ cloud}

Systems of many QCD strings have been studied since early 1990's in the context of Lund-like models of particle production. Studies of the interaction of non-overlapping strings have been done on the lattice, in a different setting. A brief review of it can be found in our papers [3, 8].

Let us consider a set of parallel and straight (unexcited) strings, called "spaghetti configuration". In experimental conditions strings have charges at the ends, moving along the beam line into positive and negative directions, but it is enough to discuss a segment of the string in the middle, of length $\delta x$. (The value of $\delta x$ is irrelevant as it appears both in the mass and the force, and cancels out.)

We will assume the string interaction to be mediated by the lightest scalar $\sigma$ - the same as in the attractive part of radial nuclear forces. For an infinite string the sigma "cloud" has a shape

$$
\frac{\left\langle\bar{q} q\left(r_{\perp}\right) W\right\rangle}{\langle W\rangle\langle\bar{q} q\rangle}=1-C K_{0}\left(m_{\sigma} \tilde{r}_{\perp}\right)
$$




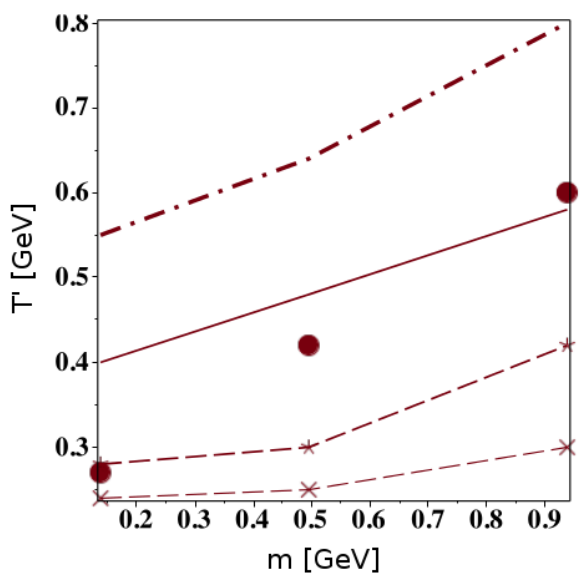

(a)

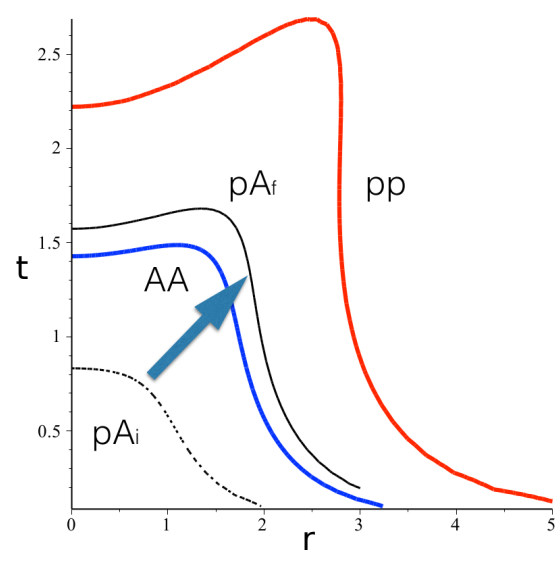

(b)

Figure 1. (a) From [6]: The slopes $T^{\prime}(m)$ (points) as a function of the particle mass $m(\mathrm{GeV})$. The solid and dash-dotted lines are hydro with the freezeout temperatures $T_{f}=0.17,0.12 \mathrm{GeV}$, respectively. The asterisks-marked dashed line is for the EPOS LHC model, diagonal crosses on the dashed line are for AMTP model: both predict the radial flow which is too small. (b) The freezeout surfaces in universal dimensionless time $t$ and radial distance $r$ coordinates. (Blue) thick solid line in the middle corresponds to central $\mathrm{AA}(\mathrm{PbPb})$ collisions, (red) thick solid line on the top to the highest multiplicity pp. Two (black) thin ones correspond to the central $\mathrm{pPb}$ case, before and after collapse compression, marked $p A_{i}, p A_{f}$, respectively. The arrow connecting them indicates the effect of a multi-string collapse.

where $K_{0}$ is the modified Bessel function and the "regularized" transverse distance is $\tilde{r}_{\perp}=\sqrt{r_{\perp}^{2}+s_{\text {string }}^{2}}$ which smooths the $2 \mathrm{D}$ Coulomb singularity $\sim \ln \left(r_{\perp}\right)$ at small $r_{\perp}$. Parameter $s_{\text {string }}$ is the "intrinsic" string width. Lattice simulations, such as [9], have found vacuum modifications due to the presence of a QCD string. We argued [3] that those data can be well described by a "sigma cloud". In Fig. 2(a) one can see our two-parameter fit to those lattice data (the sigma mass here was taken to be $m_{\sigma}=600 \mathrm{MeV}$ as an input, and the fitted "intrinsic" string width is $s_{\text {string }} \simeq 0.176 \mathrm{fm}$, consistent with other determinations such as [10]).

From the fit one can see that the main parameter of the string-string interaction (in string tension units) is numerically small, $g_{N} \sigma_{T}=\frac{\langle\sigma\rangle^{2} C^{2}}{4 \sigma_{T}} \ll 1$, so it was correctly neglected in the situations for which the Lund model has been originally invented - when only $O(1)$ strings are created.

\section{Multistring collapse}

Assuming the strings are parallel to each other, the problem can be viewed as a 2D gas of particles (in transverse plane) with unit masses at positions $\vec{r}_{i}$. The forces between each pair of strings are given by the derivative of the energy given above,

$$
\ddot{\overrightarrow{r_{i}}}=\vec{f}_{i j}=\frac{\vec{r}_{i j}}{\tilde{r}_{i j}}\left(g_{N} \sigma_{T}\right) m_{\sigma} 2 K_{1}\left(m_{\sigma} \tilde{r}_{i j}\right),
$$

with $\vec{r}_{i j}=\vec{r}_{j}-\vec{r}_{i}$ and "regularized" $\tilde{r}$. Classical molecular dynamics code follows the particle evolution in the transverse plane. In Figs. 2(c, d) we show two snapshots of one particular configuration, with $N_{s}=40$ strings at coupling $g_{N} \sigma_{T}=0.2$. The evolution consists of two qualitatively distinct parts: (i) early implosion, which converts potential energy into the kinetic one, which has its peak when fraction of the particles "gravitationally collapse" into a tight cluster; and (ii) subsequent approach to a "mini-galaxy" in virial quasi-equilibrium. In reality there is no time for the phase (ii) due to the string breaking at small $N_{s}$ or hydro explosion at large $N_{s}$.

We also calculated the mean $\sigma$ field in cylindrical geometry for a given source. An example of such calculation [8] is shown in Fig. 2(b). Two radii roughly correspond to the initial and final time. Another example of the instantaneous collective $\sigma$ field produced by the strings in the transverse plane is shown in Fig. 2(e). The white regions correspond to the values of potential smaller than $-5 \cdot 2 g_{N} \sigma_{T}\left(\mathrm{fm}^{-1}\right) \approx-400 \mathrm{MeV}$, i.e. the chiral symmetry can be completely 


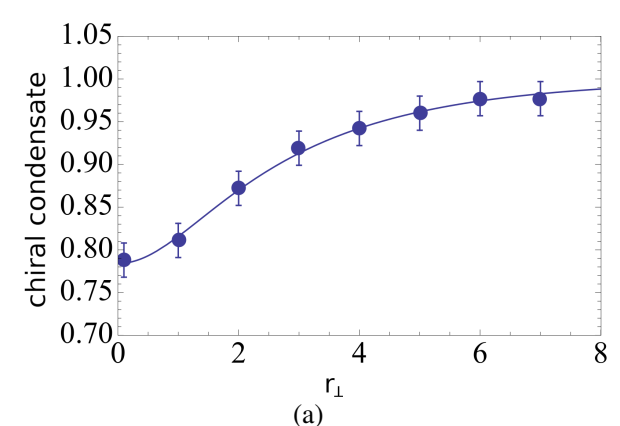

(a)

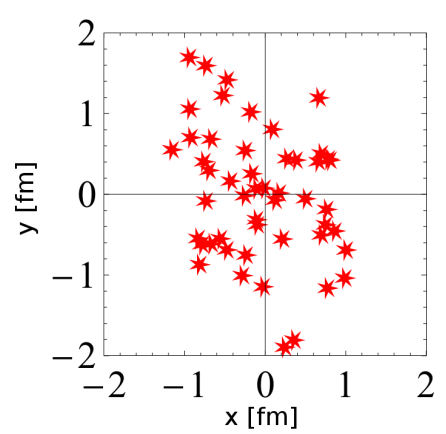

(c)

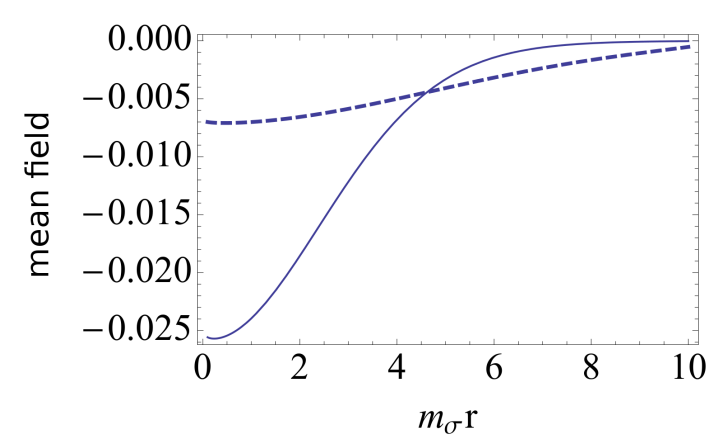

(b)

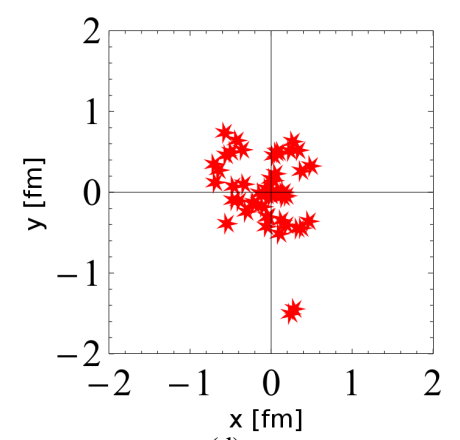

(d)

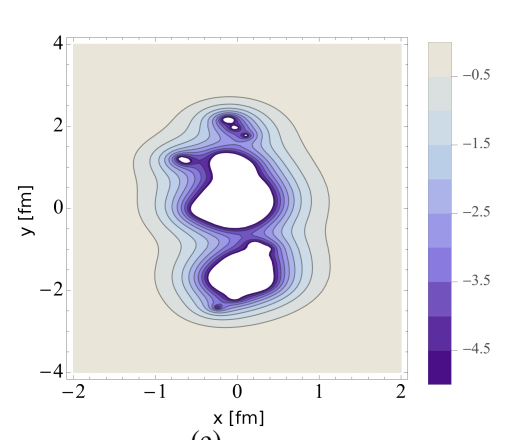

(e)

Figure 2. (a) The $\sigma$ field (normalized chiral condensate) as a function of the radial coordinate transverse to the QCD string, in lattice units. Points are from the lattice simulation [9], the curve is expression in the text with $C=0.26, s_{\text {string }}=0.176$ fm; (b) The mean $\sigma$ field versus the transverse radius (in units of inverse $m_{\sigma}$ ). The dashed and solid curves correspond to the source radii $R=1.5 \mathrm{and} 0.7 \mathrm{fm}$, respectively; (c, d) Example of a collapse in the 50 string set: two pictures correspond to the same initial configuration evolved to the times $\tau=0.1,1 \mathrm{fm} / c$, respectively. The distances are given in $\mathrm{fm}$, and $g_{N} \sigma_{T}=0.2$; (e) Instantaneous collective potential in units $2 g_{N} \sigma_{T}$ for a peripheral $A A$ configuration with $b=11 \mathrm{fm}$, $g_{N} \sigma_{T}=0.2, N_{s}=50$ at the moment of time $\tau=1 \mathrm{fm} / c$. White regions correspond to the chirally restored phase.

restored in those regions. We consider such regions as a formation of the QGP. Large gradient of this potential at its edge can cause quark pair production, similar to the Schwinger process in electric fields: one particle may flow outward and one falls into the well. Such phenomenon is a QCD analog to Hawking radiation at the black hole horizon.

Acknowledgements. This work was supported in part by the U.S. Department of Energy under Contract No. DE-FG-88ER40388.

\section{References}

[1] G. Basar, D. E. Kharzeev, H. -U. Yee and I. Zahed, Phys. Rev. D 85, 105005 (2012) [arXiv:1202.0831 [hep-th]].

[2] E. Shuryak and I. Zahed, Phys. Rev. D 89, 094001 (2014) [arXiv:1311.0836 [hep-ph]].

[3] T. Kalaydzhyan and E. Shuryak, Phys. Rev. D 90, 025031 (2014) [arXiv:1402.7363 [hep-ph]].

[4] S. Chatrchyan et al. [CMS Collaboration], EPJC 74 (2014) 2847; arXiv:1307.3442 [hep-ex]

[5] B. B. Abelev et al. [ALICE Collaboration], Phys. Lett. B 728, 25 (2014) [arXiv:1307.6796 [nucl-ex]].

[6] E. Shuryak and I. Zahed, Phys. Rev. C 88, no. 4, 044915 (2013) [arXiv:1301.4470 [hep-ph]].

[7] S. S. Gubser, Phys. Rev. D 82, 085027 (2010) [arXiv:1006.0006 [hep-th]].

[8] T. Kalaydzhyan and E. Shuryak, Phys. Rev. C 90, 014901 (2014) [arXiv:1404.1888 [hep-ph]].

[9] T. Iritani, G. Cossu and S. Hashimoto, arXiv:1311.0218 [hep-lat].

[10] P. Cea, L. Cosmai, F. Cuteri and A. Papa, [arXiv:1404.1172 [hep-lat]]. 